\title{
Ultrafast (Bio)physical and (Bio)chemical Dynamics
}

\author{
Cristina Consani, Olivier Braem, Ahmad Ajdarzadeh Oskouei, Renske M. van der Veen, \\ Amal EINahhas, Andrea Cannizzo, Gerald Auböck, Frank van Mourik, and Majed Chergui
}

\begin{abstract}
We give an overview of our recent work on ultrafast dynamics of chemical (organic dyes, metal-complexes, colloidal quantum dots), and biological (retinal and haem proteins) systems in the liquid phase, studied with a variety of ultrafast optical techniques from the infrared to the ultraviolet.
\end{abstract}

Keywords: Ultrafast dynamics

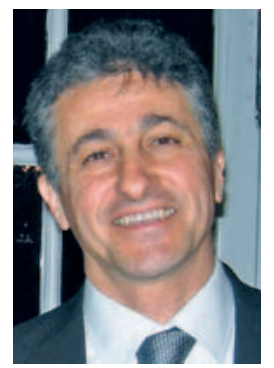

\section{Introduction}

It is now 30 years ago that the first femtosecond laser sources were developed,,$[1,2]$ which led to the research fields of femtobiology and femtochemistry. ${ }^{[3,4]}$ Several major technological and methodological developments using linear and non-linear optical phenomena have in the meantime taken place, which pushed the experimental capabilities to a level unknown before. Our laboratory is active in the development or improvement of new ultrafast methods aimed at a detailed description of photoinduced processes in the molecular condensed phase (liquid, solid and proteins) and in metallic and semiconductor nanostructured materials.

The physical phenomena we investigate include: solvation dynamics in polar and non-polar media ${ }^{[5-9]}$ and at interfaces, ${ }^{[10,11]}$ protein dynamics, ${ }^{[12-19]}$ the excited state dynamics in coordination chemistry complexes, ${ }^{[6,20-26]}$ and the dynamics of charge carriers and phonons in semicon-

${ }^{*}$ Correspondence: Prof. M. Chergui Ecole Polytechnique Fédérale de Lausanne Laboratoire de Spectroscopie Ultrarapide ISIC-FSB, station 6

$\mathrm{CH}-1015$ Lausanne

Tel.: +41216930457

E-mail: majed.chergui@epfl.ch ductor $^{[27-31]}$ and in metallic nanoparticles of different shapes and sizes. ${ }^{[32,33]}$

The experimental tools we use include femtosecond pump/continuum probe transient absorption spectroscopy, ${ }^{[15]}$ ultrafast fluorescence up-conversion spectroscopy, ${ }^{[16,17,34]}$ photon-echo spectroscopy, ${ }^{[7,8]}$ and Kerr microscopy. ${ }^{[1,35]}$ One of the highlights of our instrumentation is the extension of many of the above methodologies to the UV (below $300 \mathrm{~nm}$ ) spectral range, which is very important for biological systems as this is the region where the most abundant amino-acid residues absorb. Finally, in the last ten years or so our group pioneered optical pump/X-ray absorption spectroscopy probe techniques using picosecond and femtosecond hard X-ray pulses from a synchrotron. ${ }^{[36-38]}$ Since our X-ray activities (mostly centered at the Swiss Light Source of the Paul-Scherer-Institut, Villigen), were recently reviewed in this journal, ${ }^{[39]}$ we focus here on our optical laser-based activities in Lausanne, highlighting the tools that are, to our knowledge, quite unique world-wide and illustrating their capabilities by examples of recent results.

\section{Experimental Tools}

As mentioned above, we use a broad range of experimental methodologies, which we briefly present hereafter:

\subsection{Femtosecond-resolved Fluorescence}

Time-resolved fluorescence up-conversion is unique in reaching the ultimate resolution of a few tens of fs in fluorescence. It consists in exciting the fluorescence of the sample with an ultrashort laser pulse and mapping its time evolution by frequency mixing it (up-conversion) with a second 'gate' pulse whose time delay is tunable with respect to the pump pulse.
The up-converted signal is thus recorded as a function of pump-gate time delay. ${ }^{[17]}$ While this technique has been known for some time, the peculiarities of our set-up are: a) a broadband detection, which allows the entire fluorescence spectrum to be recorded as a function of time; b) its high sensitivity, since we can tune the laser repetition rate between 50 and $300 \mathrm{kHz}$ (typically we get $4 \mu \mathrm{J}, 800 \mathrm{~nm}$ pulse of $50 \mathrm{fs}$ width at a repetition rate of $250 \mathrm{kHz}$ ); c) its extended detection range from the mid IR $(1-2 \mu \mathrm{m})^{[30]}$ to the UV below $300 \mathrm{~nm} .{ }^{[34]}$

\subsection{Photon Echo Peak Shift (PEPS)}

Photon echo techniques are well-established tools to disentangle homogeneous from inhomogeneous contributions to spectral line shapes, and therefore to probe the fluctuations and the solvation dynamics around chromophores in proteins or solutions. Three pulse photon echo peak shift (3PEPS) experiments in the UV are implemented in our laboratory. We generate pulses at a central wavelength of 285 $290 \mathrm{~nm}$ with a bandwidth of about $4 \mathrm{~nm}$, and pulse energy of 10-20 nJ by optical non-linear techniques. The input beam is divided into three beams (1,2 and 3) of equal intensity, whose relative delay is individually controlled by two high precision flexure translation stages. The three beams are focused into the sample by a lens in a triangular configuration. ${ }^{[40,41]}$ The photon echo signal is detected in the two phasematched direction $\left(\mathrm{k}_{\mathrm{s}}=-\mathrm{k}_{1}+\mathrm{k}_{2}+\mathrm{k}_{3}\right.$, and $\mathrm{k}_{1}-\mathrm{k}_{2}+\mathrm{k}_{3}$ ) simultaneously by means of two avalanche photodiodes. For spectrally resolved detection, one of these diodes is replaced by a fiber spectrometer. The sample is a thin (typ. $200 \mu \mathrm{m}$ ) free flowing jet, in order to avoid the strong cross-phase modulation signal that would be generated in a flow-cell window. The off-resonant third order signals from the window material are typically stronger than the signal from the sample, which would make it impossible to 
measure signals at short times. By detecting simultaneously the signals in the two directions $-\mathrm{k}_{1}+\mathrm{k}_{2}+\mathrm{k}_{3}$ and $\mathrm{k}_{1}-\mathrm{k}_{2}+\mathrm{k}_{3}$, we determine the photon echo peak shift as half the delay between the maxima $\left(\mathrm{t}_{\text {max }}\right)$ of the two curves. Pulse compression of the UV pulses is optimized using dispersed detection of transient grating signals, i.e. using the setup as a transient grating FROG. ${ }^{[42]}$

\subsection{Pump-probe Techniques}

We use broadband detection in the UVVis spectral range using (two) $1 \mathrm{kHz}$ repetition rate amplified fs laser systems. The pump and probe pulses are generated from non-collinear optical parametric amplifiers (NOPA). The UV continuum probe (typically from 270 to $370 \mathrm{~nm}$ ) is generated by the principle of achromatic doubling. ${ }^{[43]}$ The time resolution (typ. $100 \mathrm{fs}$ ) is generally limited by the sample, e.g. biological samples generally require a flow cell. Dispersed detection of the probe pulses is performed using diode array detectors, or line scan CCD cameras, always with a frame-rate synchronized to the laser repetition rate, so as to measure single shot spectra.

\section{Results}

\subsection{Protein Dynamics}

Retinal proteins (rhodopsin, Bacteriorhodopsin-bR, etc.), share an active optical center that contains a retinal molecule. In rhodopsin, photoabsorption causes a cistrans isomerization, ${ }^{[44]}$ in bR the isomerization is trans-cis. In both cases, the excited state has a large dipole-moment, ${ }^{[45,46]}$ which has been suspected to play a role in the protein function. In order to understand this aspect, we investigated the response of the protein environment to the sudden excitation of this dipole with 50-fs time resolution, by probing the electro-chromism of the intrinsic tryptophan (Trp, also labeled W) absorption bands. Because of their vicinity to the retinal, the Trp residues 86 and 182 represent ideal voltmeters of the intraprotein field (Fig. 1).

We performed ultrafast transient absorption spectroscopy of wild-type bacteriorhodopsin (WT bR) and two tryptophan mutants (W86F and W182F, which lack either of the tryptophans) with visible light excitation (pump) and UV probe. Previously, ${ }^{[12,13]}$ we had demonstrated the sensitivity of the tryptophan W86F to the optically induced dipole moment of retinal. However, separating the tryptophan signal from the UV-signals of the retinal was possible only through modeling. Fig. 2 shows a systematic, quantitative direct comparison of the transient difference absorption spectra in the UV region of the three samples, so as to directly expose the

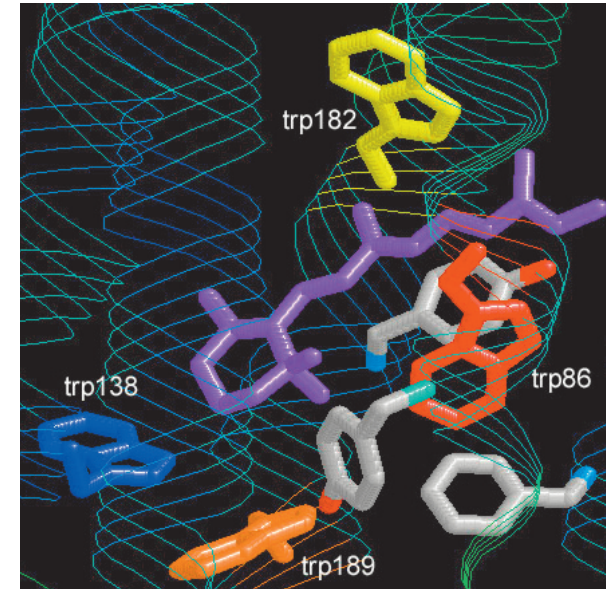

Fig. 1. Active centre of bacteriorhodopsin. The retinal chromophore is shown in purple. The Trp's (W) that were mutated are the W182 and W86 ones.

signature of each of the Trp residues. The absorption around $300 \mathrm{~nm}$ in the mutant lacking the W86 tryptophan is missing. When comparing the difference spectra of $\mathrm{W} 86 \mathrm{~F}$ with the other mutant, and the WT, it is also clear that the bleaching signal (around $275 \mathrm{~nm}$ ) of W86F is only 75\% that of the others, and this can be understood as a Stark shift of W86 (present in the WT and in W182F). A simple excitonic model describing the dipolar interaction of the retinal moiety with the two tryptophan residues (W86 and W182) allows us to reproduce the dominant features of the transient signals observed in the three samples at ultra short pump-probe delays. In particular, we show that the Trp W86 undergoes a significant Stark shift induced by the transient retinal dipole moment. The kinetics of this signal shows an instantaneous rise, followed by a decay over about $500 \mathrm{fs}$ corresponding to the isomerization time of bR. Interestingly, it does not decay back to zero, thus revealing a change in the local electrostatic environment that remains long after isomerization, in the $\mathrm{K}$ intermediate state of the protein cycle. The comparison of WT bR and W86F also leads to a revised interpretation of the overall transient UV absorption of bR.

The above UV transient absorption experiments were the seed to implementing more systematic studies of protein dynamics using Trp as a local reporter. We recently probed the coupling between the haem moiety of ferric and ferrous Horse Heart Cytochrome $\mathrm{c}$ and its single Tryptophan (Trp) residue. ${ }^{[47]}$ While energy transfer from the Trp to the haem is observed both in the decay of the Trp signal and in the delayed response of the haem, we do not observe signatures of a long-range protein response to optical excitation of the haem using Trp as a local probe. This is presumably due to the unfavourable orientation of the Trp with respect to the porphyrin plane. Changing the amount of energy deposited in the haem by a factor up to two does not affect the relaxation and cooling processes. Interesting differences are instead observed between the cooling time-scales of the two redox states, which are attributed to different haem-protein couplings.

Parallel to this work we also carried out ultrafast fluorescence up-conversion studies of the protonated Schiff base of retinal in various solvents to understand the influence of the solvent dielectric properties, viscosity and density on the conformational changes of retinal, ${ }^{[17-19]}$ showing that there is conformational heterogeneity of the excited retinal Schiff base in the solvent environment, influencing the decay but also the population channels. These conclusions also reinforce the claim that steric effects play an important role in the dynamics of the chromophore in the protein environment. A similar comparison between solvent and protein environment had also been made using fs fluorescence up-conversion in the case of the photoactive yellow protein (GFP) dynamics. ${ }^{[16]}$

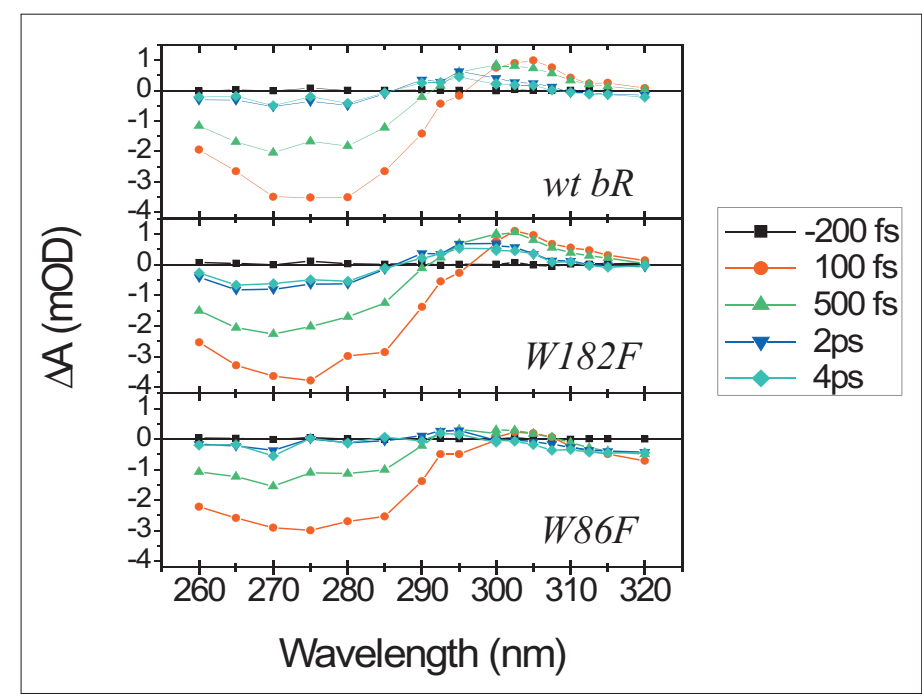

Fig. 2. UV absorption difference spectra measured at five selected delay times after excitation at 550 $\mathrm{nm}$, the spectra are constructed from single wavelength pumpprobe experiments at 20 probe wavelengths. The samples were adjusted to the same optical density at $550 \mathrm{~nm}$, and at each wavelength the signals of the three samples were acquired sequentially. ${ }^{[14]}$ 


\subsection{Solvation Dynamics}

Solvation dynamics represents the rearrangement of the solvent shell around a solute upon a change of electronic structure of the latter (Fig. 3). This modifies the field of forces within the molecule, driving its structural dynamics, but also the intermolecular field of forces, which leads to changes in the solvation shell. Because most chemical and biochemical reactions take place in solutions, the role of the solvent in hindering, enhancing or modifying reaction pathways is crucial, and this explains why the study of solvation dynamics has been so intense ever since the advent of femtosecond spectroscopy. ${ }^{[48,49]}$ In polar solvent (Fig. 3), the response is mainly dielectric, while in non-polar ones, it is mechanical as a response to changes in shape and/or size of the solute. One of the complexities of solvation dynamics has to do with the fact that it is difficult to disentangle the intra- from the intermolecular dynamics, and therefore our group combines photon echo and fluorescence up-conversion experiments, with ultrafast X-ray absorption spectroscopy[50] and detailed molecular dynamics (MD) simulations.

We implemented the first three-pulse UV photon echo experiments ${ }^{[7,8]}$ on small organic dyes in solution. The advantage of the three-pulse over the usual two-pulse methods ${ }^{[51]}$ lies in the elimination of offresonant signals, which are of special importance in the UV because of the proximity of the strong absorption bands of all solvents below $200 \mathrm{~nm}$. We measured the electronic dephasing times of the nonpolar chromophore diphenylacetylene (DPA) in ethanol and in cyclohexane polar and nonpolar solvents, respectively. Contrary to previous reports, ${ }^{[51]}$ we observed sub-100-fs electronic dephasing times for DPA in both solvents. We also identified fast dynamics of $40 \pm 10$ fs on the photon echo peak shift PEPS traces of DPA in ethanol. We demonstrated that one can exploit the fact that the bandwidth of the pulses is much less than the absorption band of the sample. In our PEPS experiments all excited state related echo signals are actually outside the probe window of the pulses, so that we effectively only measure ground-state dynamics. ${ }^{[8]} \mathrm{We}$ also observed a dependence of the PEPS asymptotic value on the temporal chirp of the pulses, and show how the chirp-dependence of the peak-shift is due to the imperfect phase-matching condition used in the PEPS technique, resolving a long-standing controversy about the latter.

As part of our strategy to use aromatic amino acids as sensors of intraprotein dynamics, we also implemented UV fluorescence up-conversion studies of tryptophan. Due to the near degeneracy of its two low- est excited states, $\mathrm{L}_{\mathrm{a}}$ and $\mathrm{L}_{\mathrm{b}}$, the initial excited state dynamics is rather complex and not fully resolved so far. We therefore started a detailed investigation of Trp in aqueous solutions, ${ }^{[9]}$ combining fluorescence up-conversion in both polychromatic and single wavelength polarization-dependent detection modes in the 300-480 $\mathrm{nm}$ range, with MD simulations. We measured the time evolution of the Stokes shift, bandwidth, and anisotropy from tens of femtoseconds to picoseconds. These observables contain signatures of both intra-molecular and solvent-molecule dynamics, which we disentangled with the help of non-equilibrium molecular dynamics simulations. Fig. 4 shows a $2 \mathrm{D}$ time-wavelength plot of the emission probed with parallel and perpendicular polarization (with respect to the excitation pulse), panels $\mathrm{C}$ and $\mathrm{D}$ present the anisotropy of the emission. The latter shows a fast shift to longer wavelengths, the center of mass of the emission spectrum shifts by $>1500 \mathrm{~cm}^{-1}$ in the first 3 ps. The time dependence actually was very well reproduced by molecular dynamics simulations giving dominant decay components of $160 \mathrm{fs}$ and $1 \mathrm{ps}$. These times are attributed to collective motions and independent diffusion of several proximal water molecules. The MD simulations also exposed a sub-10 fs decay that accounts for $\sim 1700 \mathrm{~cm}^{-1}$ of the total Stokes shift of 3800

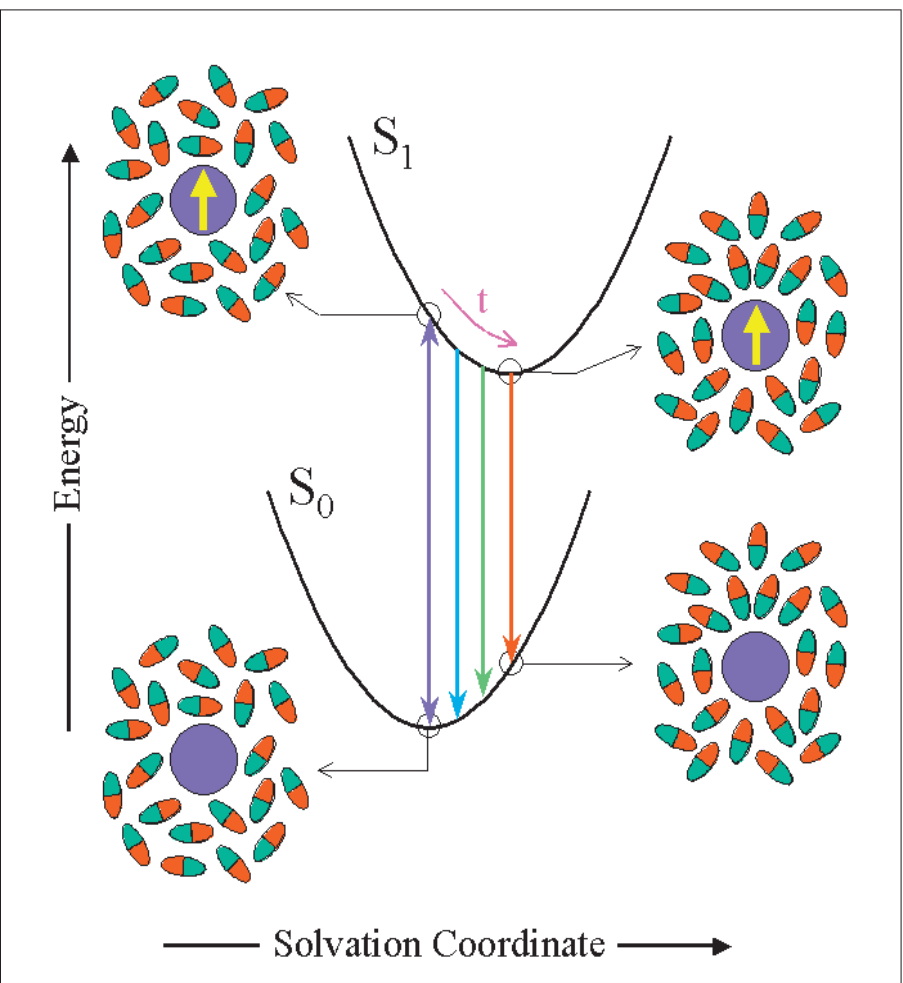

Fig. 3. The process of solvation dynamics in a polar solvent. Excitation of a dye molecule (purple) generates a dipole to which the solvent molecules respond by minimizing the free energy along some solvent coordinate. Once the new equilibrium configuration is reached the dye molecule can emit. In the course of solvent rearrangement the fluorescence (downward arrows) can be followed by fluorescence up-conversion techniques, which measure the dynamical Stokes shift.

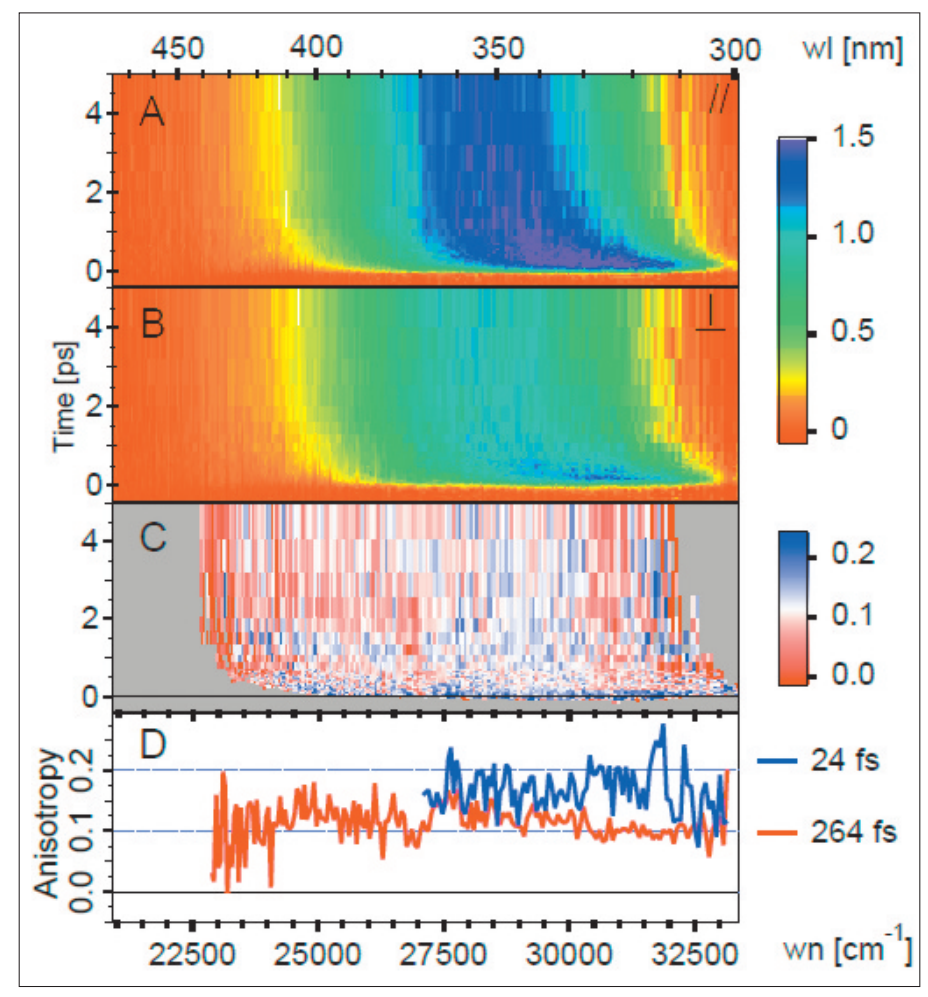

Fig. 4. Time-wave number plots of the fluorescence spectrum of tryptophan in water in the first $5 \mathrm{ps}$, for parallel $(A)$ and orthogonal $(B)$ polarization. (C) Time-wavenumber plot of the anisotropy over the first 5 ps. The black horizontal line corresponds to time zero. Selected anisotropy spectra at $24 \mathrm{fs}$ and 264 fs are shown in panel D. ${ }^{[9]}$ Reprinted with permission from J. Phys. Chem A 2010, 114, 9034. Copyright 2010 American Chemical Society. 
$\mathrm{cm}^{-1}$. The origin of this component is intermolecular, i.e. due to solvation. It comes from the inertial motion of the water molecules and is associated with their independent rotational motion. Finally, from the anisotropy we extracted an equilibration time of 40 fs between the $\mathrm{L}_{\mathrm{a}}$ and $\mathrm{L}_{\mathrm{b}}$ states. This was reported before for tryptophan in rubredoxin ${ }^{[52]}$ but our improved time resolution, combined with the MD study, gives a more solid estimate for this time.

\subsection{Ultrafast Dynamics of Metal- based Complexes}

Closely related to our X-ray experiments, $[36,37,39,53]$ but also to our studies on hemoproteins is the investigation of metalbased molecular complexes in solution. These are also of importance for several applications such as solar energy, photocatalysis, magnetic data storage, etc. Many of these metal-complexes have characteristic absorption bands due to singlet metal-toligand charge transfer ( ${ }^{1}$ MLCT) states, which are the doorway states upon optical excitation, leading to charge transfer, spin changes and structural changes, which we studied in recent years using the various ultrafast optical and X-ray techniques of our group. The following systems/processes have been investigated:

\subsubsection{Ruthenium-based Complexes}

We investigated the model compound $\left[\mathrm{Ru}(\mathrm{bpy})_{3}\right]^{2+}$ using ps X-ray absorption spectroscopy, ${ }^{[54]}$ fs optical transient absorption ${ }^{[20]}$ and fs broadband fluorescence up-conversion. ${ }^{[55]}$ Fig. 5 shows the timewavelength plot of the emission of the

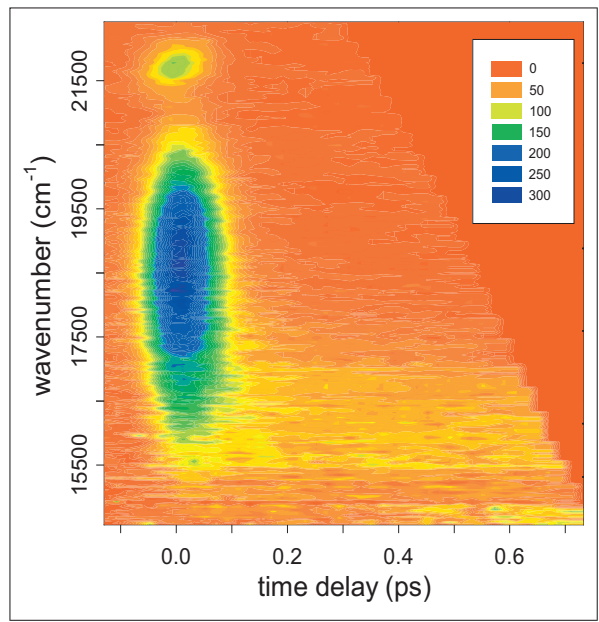

Fig. 5. Time-wavenumber plot of time resolved luminescence of aqueous $\left[\mathrm{Ru}(\mathrm{bpy})_{3}\right]^{2+}$ under excitation at $400 \mathrm{~nm}\left(25000 \mathrm{~cm}^{-1}\right)$. The signal at $\sim 21600 \mathrm{~cm}^{-1}$ is the Raman line of water. The ${ }^{1}$ MLCT fluorescence appears within the pump pulse centred at $18500 \mathrm{~cm}^{-1}$. The yellow strip centred at $16300 \mathrm{~cm}^{-1}$ corresponds to the ${ }^{3}$ MLCT phosphorescence. ${ }^{[55]}$ Reprinted with permisson from Angew. Chem. Int. Ed. 2006, 45, 3174. Copyright John Wiley and Sons 2006. system where the short-lived fluorescence centered at $17500 \mathrm{~cm}^{-1}(540 \mathrm{~nm})$ is due to emission of the ${ }^{1}$ MLCT state, while the long-lived emission that appears as a faint strip centred around $16300 \mathrm{~cm}^{-1}(620 \mathrm{~nm})$ is the well-known ${ }^{3} \mathrm{MLCT}$ phosphorescence. Here we would like to highlight the performance of our set up since the pure radiative lifetime of the phosphorescence is about $10 \mu \mathrm{s}$, which implies a detection efficiency of $1: 10^{7}$. From the kinetic traces extracted from Fig. 4, we derive an ultrafast ${ }^{1}$ MLCT${ }^{3}$ MLCT intersystem crossing (ISC) of $\leq 30$ fs! This ultrafast ISC has also been found for the RuN3 and RuN719 dyes, ${ }^{[56]}$ which are those used in dye-sensitized solar cells. We also carried out fs fluorescence studies of $\mathrm{RnN7} 719$ on $\mathrm{TiO}_{2}$ (electron injecting) and $\mathrm{Al}_{2} \mathrm{O}_{3}$ (non-injecting) substrates, and our analysis shows that the main factor limiting the initial electron injection from non-thermalized levels of the dye into the substrate ${ }^{[57]}$ is the intramolecular vibrational redistribution (IVR). This also led us to estimate an injection time of $<10 \mathrm{fs}$.

\subsubsection{Iron(ii)-based Complexes}

These so-called spin cross-over complexes have attracted much interest in the past 20 years or so due to their ability to undergo $S=0$ to $S=2$ spin changes under the effect of pressure, temperature or photoexcitation. ${ }^{[58,59]}$ We focused our studies on the smallest member of the family $\left[\mathrm{Fe}(\mathrm{bpy})_{3}\right]^{2+}$ (Fig. 6) which can undergo the $S=0$ to $S=2$ spin transition only under the effect of light excitation. Our optical and X-ray studies, ${ }^{[22,23,60-62]}$ have shown a similar sub-30 fs ${ }^{1}$ MLCT- ${ }^{3}$ MLCT ISC as in the Ru-complex

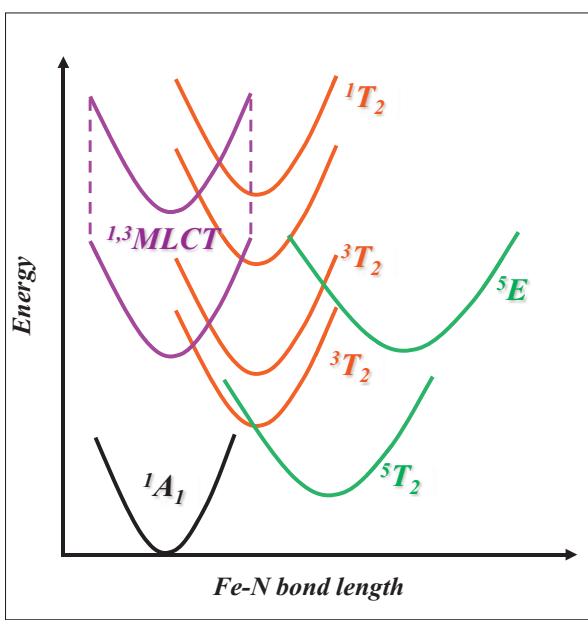

Fig. 6. Potential curve diagramme of $\left[\mathrm{Fe}(\mathrm{bpy})_{3}\right]^{2+}$ along the Fe- $\mathrm{N}$ coordinate, which is the main reaction coordinate of the spin crossover. Excitation of the MLCT states leads to ultrafast population of the lowest lying metalcentred ${ }^{5}$ T state. ${ }^{[22]}$

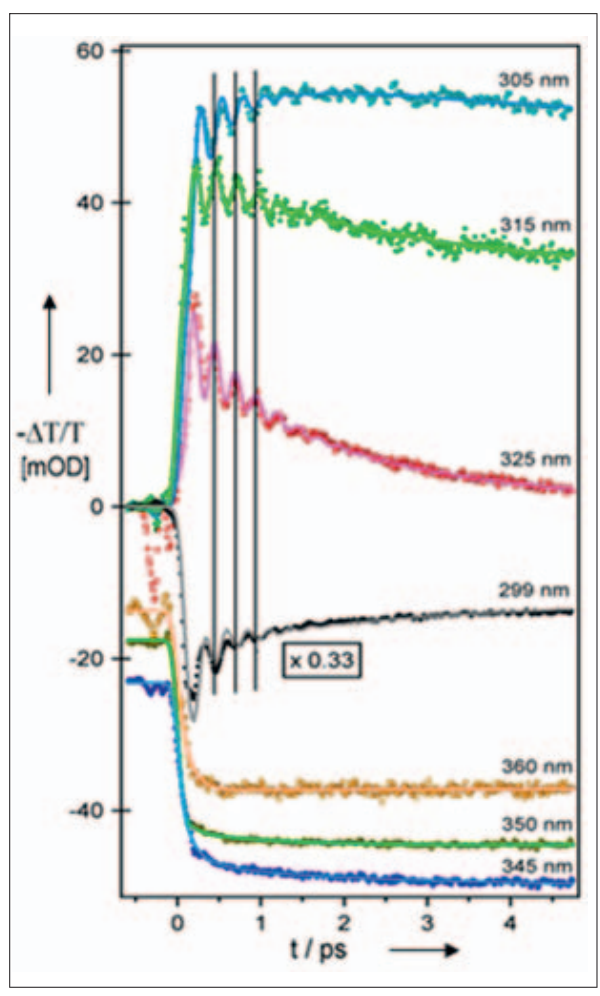

Fig. 7. Kinetic traces at different UV probe wavelengths of aqueous $\left[\mathrm{Fe}(\mathrm{bpy})_{3}\right]^{2+}$ excited at $400 \mathrm{~nm}$. The probe wavelengths cover the quintet excited state absorption (305, 315 and $325 \mathrm{~nm})$ and the bleach regions (299, 345, 350 and $360 \mathrm{~nm}$ ) of the spectrum. Solid lines represent global fits. The vertical lines show the phase shift of $\sim \pi$ of the oscillations between the blue most and the red most probe wavelengths in the ESA region. For sake of clarity time-traces at $\lambda>340 \mathrm{~nm}$ have been vertically shifted and the $299 \mathrm{~nm}$ one is scaled to $1 / 3 .^{[23]}$ Reprinted with permisson from Angew. Chem. Int. Ed. 2009, 48, 7184. Copyright John Wiley and Sons 2009.

but also for the first time, that the MLCT states decay directly into the high lying levels of the lowest metal-centered quintet $\left({ }^{5} \mathrm{~T}\right)$ state, thus bypassing several intermediate metal-centered singlet and triplet $\mathrm{T}$ states (Fig. 6). This leads to a vibrationally hot quintet state, whose rise, cooling and decay we could follow by deep UV transient absorption spectroscopy, since the quintet state has high lying absorption bands. ${ }^{[23]}$ Fig. 7 illustrates our capability at capturing the dynamics and relaxation in the quintet state using our visible pump/UV continuum probe set up. The rich wave packet dynamics therein appearing is due to the lowest frequency mode of the molecule, which is impulsively excited by the ultrafast nonadiabatic MLCT-5 T curve crossing event. The combination of the optical visible and UV transient absorption studies, the fs fluorescence ones, and the ps/fs X-ray studies allowed us to fully resolve the photocycle of the system. ${ }^{[22]}$ The understanding of the photophysics of Fe-polypyridine complexes is also shedding light on the electronic 
and vibrational dynamics occurring in Ironbased porphyrins and hemoproteins.

\subsubsection{Rhenium(i)-halogen Carbonyl- bipyridine Complexes}

The above-mentioned issues of ultrafast intersystem crossing in $\mathrm{M}(\mathrm{bpy})_{3}(\mathrm{M}=\mathrm{Ru}$, $\mathrm{Fe})$ complexes, as well as the questions of the ensuing structural changes led us to investigate the photophysics of $\operatorname{Re}(\mathrm{X})$ $(\mathrm{CO})_{3}$ (bpy) complexes, which offer the advantage of substituting one of the ligands by a halogen atom $(\mathrm{X})$. In these complexes the Re-halogen moiety is strongly mixed, reaching a 50:50 mixture of orbitals for the case of iodine. The electron transfer process is therefore an MLLCT (metalligand-to-ligand-CT) one. We carried detailed fs optical fluorescence and transient absorption studies, which were combined with extensive quantum-chemical calculations. ${ }^{[21,24,25,63]}$ Some of the remarkable findings of this study are the fact that the ultrafast ISC from singlet to triplet is significantly longer that in the above tris-bipyridine complexes, even though Re has a larger spin-orbit (SO) constant than $\mathrm{Ru}$ or $\mathrm{Fe}$. Also, the ISC time increases ${ }^{[21]}$ in the series Cl-Br-I (from $\sim 100$ to $150 \mathrm{fs}$ ), which is contrary to the expected trends considering the SO constant of the halogen atom. We also found that the ISC time scales directly with the vibrational period of the Re-halogen bond, which strongly points to a structural basis for the ISC: the system explores its landscape in order to undergo the spin flip. The other remarkable finding of these studies is a solvent dependence of the electron transfer process. Indeed, our results show a prompt electron transfer upon excitation of the MLLCT state followed by an additional component of several ps, which cannot be attributed to cooling. We believe that solvent molecules intercalated between the ligands may play an important role in the ET process. Such intercalation of solvent molecules was found in quantum mechanics/molecular mechanics simulations ${ }^{[64,65]}$ and confirmed by recent static soft X-ray experiments. ${ }^{[53]}$

\subsubsection{Diplatinum-based Molecular Complexes}

Binuclear $d^{8}-d^{8}$ molecular complexes of rhodium, iridium and platinum bridged by various ligands are interesting because of the photochemical and photophysical properties arising from the lowest singlet and triplet ${ }^{1,3} \mathrm{~A}_{2 \mathrm{u}}\left(\mathrm{d} \sigma^{*} \rightarrow \mathrm{p} \sigma\right)$ excited states (Fig. 8 for the case of the $\left[\mathrm{Pt}_{2}\left(\mathrm{P}_{2} \mathrm{O}_{5} \mathrm{H}_{2}\right)_{4}\right]^{4-}$ complex). The promotion of an electron from the antibonding $\mathrm{d} \sigma^{*}$ orbital $\left(\mathrm{d}_{z}^{2}\right.$-derived, $z$-axis along $\mathrm{Pt}-\mathrm{Pt}$ ) into the bonding $\mathrm{p} \sigma$ orbital ( $\mathrm{p}_{z}$-derived) leads to formation of a Pt-Pt bond and a contraction of the $\mathrm{Pt}-\mathrm{Pt}$ distance, which characterizes the structure of the ${ }^{1,3} \mathrm{~A}_{2 \mathrm{u}}$ states. The remark-

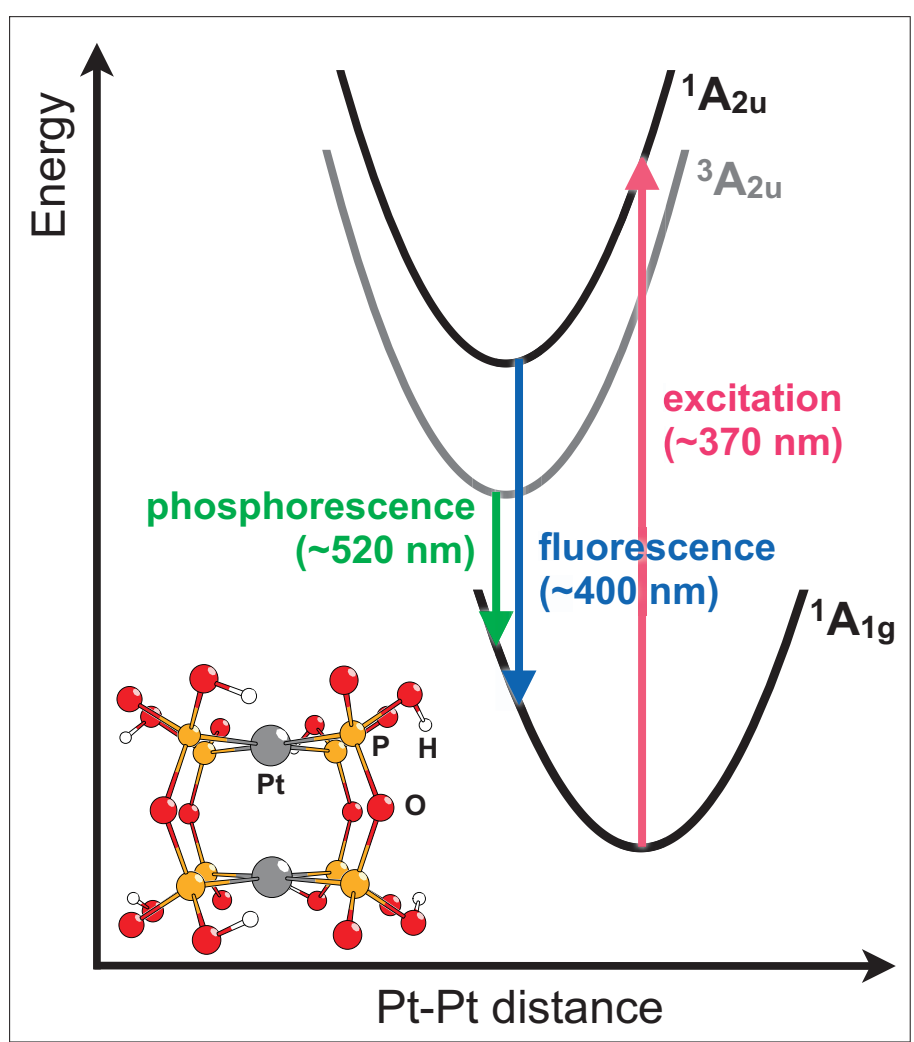

Fig. 8. Simplified potential energy scheme of the ${ }^{1} A_{1}$ ground state and ${ }^{1,3} A_{2 u}$ lowest-excited states of PtPOP. Note that the ${ }^{1} A_{2 u}$ and ${ }^{3} A_{2 u}$ potential curves are vertically parallel. The optical transitions are indicated by vertical arrows. The molecular structure of PtPOP is shown on the left. In our experiments we probed both the transient absorption and the ultrafast fluorescence of the system upon excitation of the singlet state. ${ }^{[26]}$ Reprinted with permisson from J. Am Chem. Soc. 2011, 133, 305. Copyright 2011 American Chemical Society.

able feature of this molecule is the fact that both its ground and its excited (singlet and triplet) states are highly harmonic and well separated in energy. They offer an ideal system to investigate the solvent effects on the vibrational relaxation, the dephasing of vibrational wave packets and the intersystem crossing. After characterizing the triplet excited state structure by ps XAS, ${ }^{66]}$ we carried out detailed fs fluorescence and optical transient absorption studies, ${ }^{[26]}$ which revealed rich dynamics.

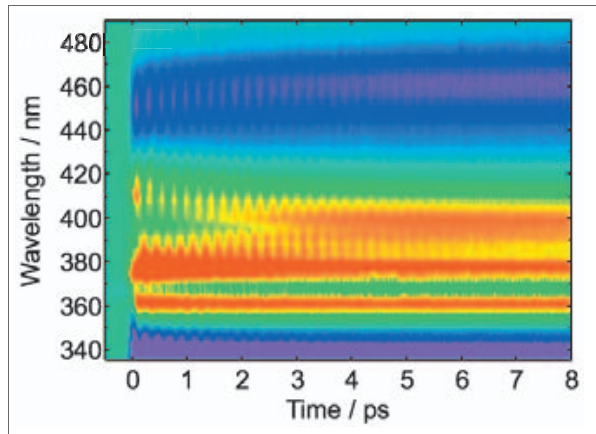

Fig. 9. Time-wavelength plot of the transient absorption of PtPOP in solution showing the ground state bleach (red region around 370 $\mathrm{nm}$ ), but also stimulated emission contributions (orange and yellow) and excited state absorption (blue) of the singlet excited state. Note the rich wave packet pattern due to oscillations of the Pt-Pt bond in the singlet excited state, but also in the ground state, which is excited by impulsive Raman scattering. ${ }^{[26]}$ Reprinted with permisson from J. Am. Chem. Soc. 2011, 133, 305. Copyright 2011 American Chemical Society.
Indeed, fs excitation of the singlet state leads to an impulsive Pt-Pt bond forming and the creation of vibrational wave packets. In addition, impulsive Raman efficiently produces wave packets in the ground-state, which can be distinguished from those in the excited state by their longer period: 281 fs for the ground-state and $224 \mathrm{fs}$ for the excited state oscillation period (Fig. 9). One of the main findings of this work is that the decay of coherence is slower than the vibrational relaxation due to the efficient transfer of coherence among vibronic levels. This implies that the decay in PtPOP does not conform to the optical Bloch picture: at the microscopic level, energy dissipation to the solvent occurs such that the coherence is preserved to a large extent. We conclude that a direct Pt-solvent energy dissipation channel accounts for the vibrational cooling in the singlet state. The ISC time (10-30 ps) from the ${ }^{1} \mathrm{~A}_{2 \mathrm{u}}$ to the ${ }^{3} \mathrm{~A}_{2 \mathrm{u}}$ state is solvent dependent and is remarkably long compared to all above-mentioned complexes if one considers that the system contains two heavy atoms. It is also significantly longer than that of monoPt complexes we measured by fluorescence up-conversion. ${ }^{[67]} \mathrm{We}$ concluded that the slow ISC stems from the particular structure, energetics, and symmetry of the molecule, which would cast it in a rational framework with the above-mentioned complexes. It is induced by spin-vibronic coupling with a higher-lying triplet state and/or (transient) symmetry breaking in the ${ }^{1} \mathrm{~A}_{2 \mathrm{u}}$ excited state. 


\subsection{Carrier Dynamics in Semiconductor and Metallic Nanoparticles (NPs)}

The study of the ultrafast carrier dynamics in nanoparticles is interesting considering their strong potential for applications in optoelectronics, imaging, plasmonics, etc. In addition, because of their engineerable properties, their optical response can be tuned by changing their chemical composition, their size and their shapes. We recently investigated both semi-conductor and metallic nanoparticles using a combination of static, ultrafast fluorescence up-conversion and transient absorption spectroscopies. A brief outline of the main results is given thereafter:

\subsubsection{Colloidal Semi-conductor Nanoparticles}

We performed femtosecond photoluminescence (PL) studies of $\mathrm{CdSe}^{[28]}$ and $\mathrm{PbSe} \mathrm{NPS}^{[30,68]}$ using our polychromatic detection. Aside from conflicting views about the relaxation cascades of both electron and hole, the identification of multi-excitonic states was unclear because only transient absorption studies had so far been carried out, which complicate the assignment of spectral features due to overlapping contributions such as excited state absorption, stimulated emission and ground state bleach. Steady state photoluminescence (PL) excitation spectroscopy of CdSe NPs demonstrated that the intradot relaxation proceeds first to the lowest excited state(s) from where luminescence takes place, pretty much like in large molecules. ${ }^{[69]}$ This rules out the often invoked quenching of higher levels by surface effects. Under moderately high fs excitation densities we observed the formation and dynamics of biexitons and triexcitons in CdSe NPs. Contrary to earlier reports, all results could be understood without invoking the presence of charged particles. The dynamics of single excitons in PbSe NPs was also studied by fs PL up-conversion implemented in the near-IR for the first time (Fig. 10), and it was found to be similar to the case of $\mathrm{CdSe}$, despite the high confinement in the former. The early-time spectra are characterized by emission from several low lying excited states. The kinetics point to a fast sequential cascade process between excited states (Fig. 10) governed by energy gaps, indicating the presence of additional dark states. The first direct measurement of the lowest excited states Huang-Rhys factors excludes that a

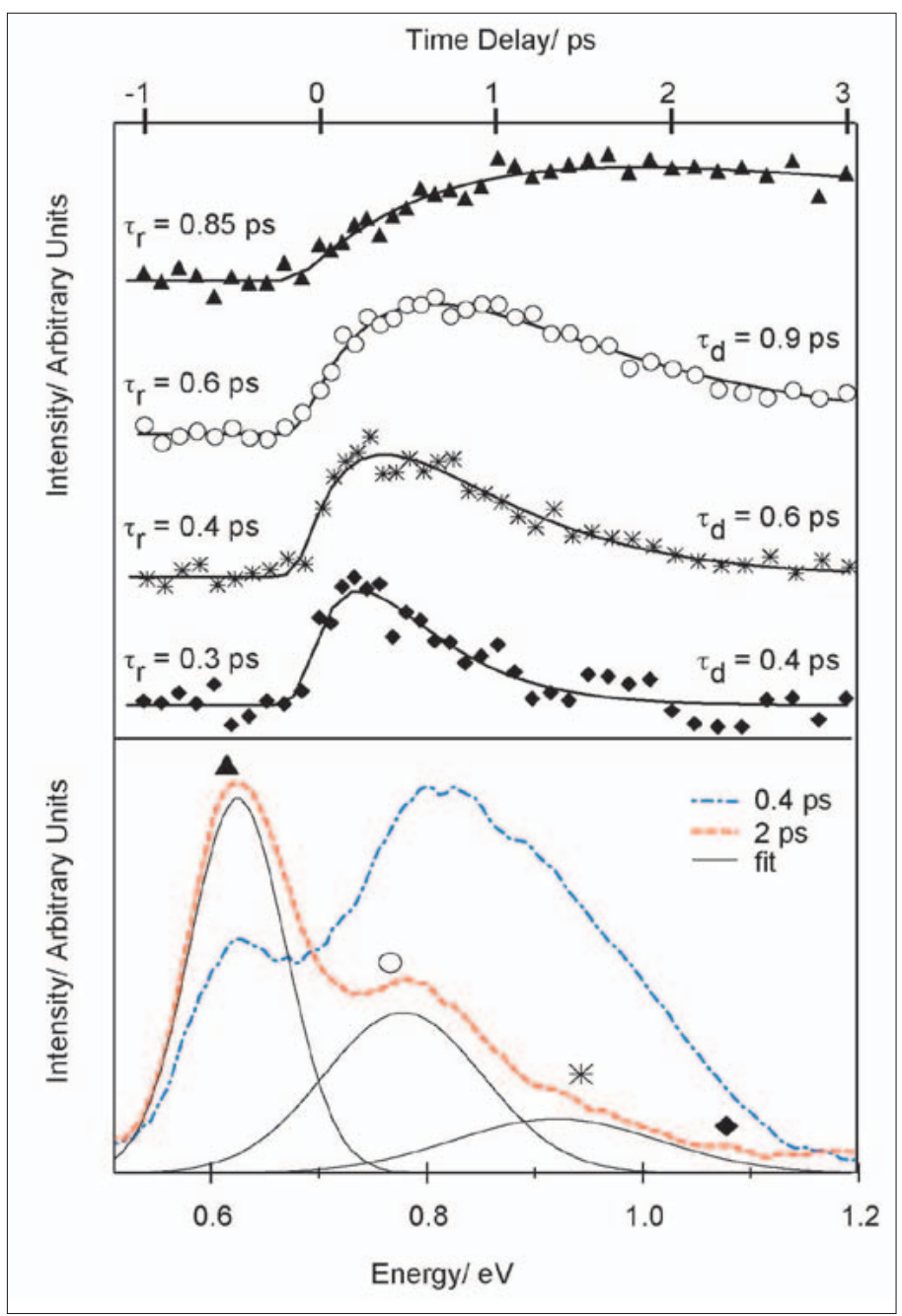

Fig. 10. (Bottom) ultrafast infrared photoluminescence of colloidal $\mathrm{PbSe}$ nanoparticles at two different time delays, as well as fit of the 2 ps spectrum showing emission from higher lying states. (Upper) Kinetic traces at different energies (marked by the symbols in lower panel, along with their corresponding biexponential fits (solid lines). The rise $(\tau)$ and decay $\left(\tau_{d}\right)$ times used for the fits are shown in the figure. From top to bottom, the traces are representative, respectively, of the dynamics of the three bands (lower panel) and of higher energy contributions. Traces have been normalized and offset for clarity. The traces illustrate clearly the stepwise cascading from higher to lower lying states. ${ }^{[30]}$ strong electron-phonon coupling mediates the intra band relaxation in $\mathrm{PbSe}$.

To address a widely disputed issue on the nature of the longer electron-recombination time-scales, we performed ns PL decay measurements of CdSe NPs over several decades of intensities and times, and as a function of size, shape and temperature. ${ }^{[29,70]}$ We proposed a model for the multi-exponential decay kinetics, and their temperature dependence, in which a major role is played by the now well-established presence of a distribution of large groundstate dipole moments in CdSe NPs. ${ }^{[31]}$ By two-photon excitation within the bandgap region we showed that there is a link between the ground-state dipole moment and the excited-state decay. The stochastic nature of the magnitude of the dipole moment results in a complex temperature dependence. Contrary to studies that ascribe non-radiative decay processes to surface states/traps, the mechanism we propose considers the intrinsic states at the band gap, described within the effective mass approximation models. Surface effects are mediated by the ground-state dipole moments, which in turn perturb the intrinsic states.

\subsubsection{Metallic NPs}

The dynamics of charge carriers in metallic NPs is very different to that of semiconductor ones due to the absence of a band gap. Under ultrashort laser excitation the hot electron gas in bulk metals relaxes on ultrafast time scales (few to tens of fs) by transferring its energy to the lattice. The efficiency of this transfer depends on the electron-phonon coupling but in most cases it leads to an impulsive excitation of the low-frequency acoustic phonon modes, which show up as coherent oscillations in transient absorption or reflectivity measurements. ${ }^{[71]}$ In NPs the type and frequency of the phonons depends on their shape and size. We carried out a systematic fs transient absorption study upon plasmon resonance excitation ${ }^{[33]}$ of triangular silver $\mathrm{NP}$ (typ. $70 \mathrm{~nm}$ long and $<8 \mathrm{~nm}$ thick) in solution, which were prepared in-house through a highly size- and shape-selective photochemical synthesis. ${ }^{[32]} \mathrm{We}$ identified two low frequency totally symmetric vibrations of the NP (Fig. 11) in full agreement with a variational elastodynamic model that is also presented. From the analysis of the phase we concluded that thermal expansion and electron pressure, respectively, are responsible for the excitation of the two vibrations.

\section{Future Prospects}

Our broadband fluorescence up-conversion is being heavily used to investigate the ultrafast dynamics in a wide range of 


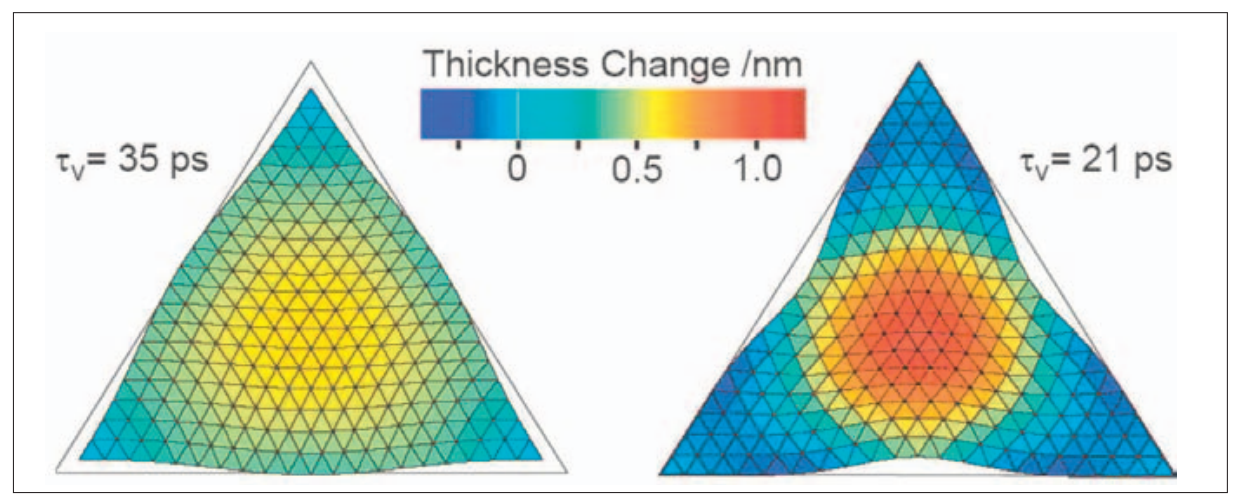

Fig. 11. Normalized displacements of the two lowest frequency $A_{1 \mathrm{~g}}$ vibrational modes calculated for a triangular silver plate with edge $70 \mathrm{~nm}$ and thickness $8 \mathrm{~nm}$ and using macroscopic parameters for the Young modulus and the density. ${ }^{\left[{ }^{[3]}\right.}$ Reprinted with permisson from Nano Letters, 2006, 6, 7. Copyright 2006 American Chemical Society.

different systems, and studies are being pursued on metal-complexes (with osmium, copper, iridium and platinum), but also on biological system both in the UV and the visible. ${ }^{[47,72]}$ In parallel UV photon echo techniques have been implemented to study the solvation dynamics of Trp in solution, ${ }^{[73]}$ for complementing the UV fluorescence up-conversion studies, ${ }^{[9]}$ demonstrating its full capabilities.

The development of UV transient absorption and UV photon echo techniques in our group is also motivated by our drive to develop UV multidimensional spectroscopy. Two-dimensional (2D) spectroscopy is an analogue to the optical domain of NMR techniques. It was first developed 10 years ago in the IR region, ${ }^{[74-77]}$ with the advantages that the time resolution is much higher than in NMR and the coupling between vibrational dipoles is also significantly higher than between magnetic dipoles in NMR, so that correlations can be monitored over larger distances. Pushing further towards shorter wavelengths switches the attention to monitoring the couplings between electronic dipoles, which are at least an order of magnitude larger than the vibrational ones, allowing for the probing of interactions over even larger distances. Moreover, a significant improvement in time resolution can be achieved. The implementation of electronic 2D was first achieved by Fleming and co-workers ${ }^{[7-80]}$ in the visible part of the spectrum. Reaching the UV in the region of the amino-acid absorption bands opens a unique opportunity to investigate the couplings and the cross-talk between them in the course of a biological function.

We have recently achieved the construction of a multidimensional optical experiment around a new versatile laser system. The laser produces pulses with the characteristics of previous $1 \mathrm{kHz}$ repetition rate amplified laser systems, but at a repetition rate of $10-50 \mathrm{KHz}$. The high
[1] R. L. Fork, B. I. Greene, C. V. Shank, Appl. Phys. Lett. 1981, 38, 671

[2] R. L. Fork, C. H. B. Cruz, P. C. Becker, C. V. Shank, Optics Lett. 1987, 12, 483.

[3] J. Martin, M. H. Vos, Ann. Rev. Biophys. Biomol. Struct. 1992, 21, 199.

[4] A. Zewail, Angew. Chem. Int. Ed. 2000, 39, 2586.

[5] P. Larregaray, A. Cavina, M. Chergui, Chem Phys. 2005, 308, 13.

[6] V. T. Pham, W. Gawelda, Y. Zaushitsyn, M Kaiser, D. Grolimund, S. L. Johnson, R. Abela, C. Bressler, M. Chergui, J. Am. Chem. Soc. 2007, 129, 1530

[7] A. A. Oskouei, O. Braem, A. Cannizzo, F. van Mourik, A. Tortschanoff, M. Chergui, J. Mol. Liquids 2008, 141, 118.

[8] A. A. Oskouei, A. Tortschanoff, O. Braem, F. van Mourik, A. Cannizzo, M. Chergui, J. Chem. Phys. 2010, 133, 064506.

[9] O. Braem, A. A. Oskouei, A. Tortschanoff, F van Mourik, M. Madrid, J. Echave, A. Cannizzo, M. Chergui, J. Phys. Chem. A 2010, 114, 9034.

[10] A. Kornherr, A. Tortschanoff, E. PortuondoCampa, F. van Mourik, M. Chergui, G. Zifferer, Chem. Phys. Lett. 2006, 430, 375.

pulse energy $(0.5 \mathrm{~mJ})$ allows a wide range of frequency-conversion techniques to be performed to generate ultrashort pulses over the UV-Vis range. By applying achromatic doubling we produce a broadband UV continuum that extends from $250 \mathrm{~nm}$ to $350 \mathrm{~nm}$ and has suitable pulse energy for $2 \mathrm{D}$ experiments. The laser system was specially designed to minimize shot-toshot fluctuations (the fundamental output has $<0.1 \%$ RMS noise), which is very important for experiments that require data averaging to obtain a good $\mathrm{S} / \mathrm{N}$ for small signals (typical for biological samples). To match the capabilities of the laser-system we developed a special multi-channel detection system capable of measuring single shot spectra of probe (and reference) pulses (for dispersed pump-probe and 2D spectroscopies). This system will allow us to fully exploit the increased repetition rate, which will translate into a decrease in the data-accumulation time of some of the experiments described above by more than order of magnitude.

In summary the combination of ultrafast X-ray absorption spectroscopy, UV optical fluorescence and UV transient absorption spectroscopy and the upcoming implementation of UV multidimensional spectroscopy allows us to harness the entire sequence of structural events of a (bio)chemical reaction from the active center to the outskirts of the protein, while at the same time delivering information about the photoinduced electronic structure changes and the correlations between chromophores.

\section{Acknowledgments}

The authors are grateful to the Swiss Science Foundation for long-standing support, more recently via the NCCR MUST, the Sekretariat für Bildung und Forschung via the COST D35 action and the European Science Foundation DYNA network.

Received: June 24, 2011
[11] E. Portuondo-Campa, A. Tortschanoff, F. van Mourik, M. Chergui, Chem. Phys. 2007, 341, 11.

[12] S. Schenkl, F. van Mourik, G. van der Zwan, S. Haacke, M. Chergui, Science 2005, 309, 917.

[13] S. Schenkl, F. van Mourik, N. Friedman, M Sheves, R. Schlesinger, S. Haacke, M. Chergui, Proc. Nat. Acad. Sci. USA 2006, 103, 4101.

[14] J. Leonard, E. Portuondo-Campa, A. Cannizzo, F. van Mourik, G. van der Zwan, J. Tittor, S. Haacke, M. Chergui, Proc. Nat. Acad. Sci. USA 2009, 106, 7718

[15] J. Helbing, L. Bonacina, R. Pietri, J. Bredenbeck, P. Hamm, F. van Mourik, F. Chaussard, A. Gonzalez-Gonzalez, M. Chergui, C. Ramos-Alvarez, C. Ruiz, J. Lopez-Garriga, Biophys. J. 2004, 87, 1881

[16] M. Vengris, M. A. van der Horst, G. Zgrablic, I. H. M. van Stokkum, S. Haacke, M. Chergui, K. J. Hellingwerf, R. van Grondelle, D. S. Larsen, Biophys. J. 2004, 87, 1848.

[17] G. Zgrablic, K. Voitchovsky, M. Kindermann, S. Haacke, M. Chergui, Biophys. J. 2005, 88, 2779.

[18] G. Zgrablic, S. Haacke, M. Chergui, Chem, Phys, 2007, 338, 168.

[19] G. Zgrablic, S. Haacke, M. Chergui, J. Phys. Chem. B 2009, 113, 4384.

[20] A. N. Tarnovsky, W. Gawelda, M. Johnson, C. Bressler, M. Chergui, J. Phys. Chem. B 2006, 110, 26497.

[21] A. Cannizzo, A. M. Blanco-Rodriguez, A el Nahhas, J. Sebera, S. Zalis, A. Vlcek, M. Chergui, J. Am. Chem. Soc. 2008, 130, 8967.

[22] A. Cannizzo, C. J. Milne, C. Consani, W. Gawelda, C. Bressler, F. van Mourik, M. Chergui, Coord. Chem. Rev. 2010, 254, 2677.

[23] C. Consani, M. Premont-Schwarz, A. ElNahhas, C. Bressler, F. van Mourik, A. Cannizzo, M. Chergui, Angew. Chem. Int. Ed. 2009, 48, 7184.

[24] A. el Nahhas, A. Cannizzo, F. van Mourik, A M. Blanco-Rodriguez, S. Zalis, A. Vlcek, M. Chergui, J. Phys. Chem. A 2010, 114, 6361 .

[25] A. El Nahhas, C. Consani, A. M. BlancoRodriguez, K. M. Lancaster, O. Braem, A Cannizzo, M. Towrie, I. P. Clark, S. Zalis, M. Chergui, A. Vlcek, Inorg. Chem. 2011, 50, 2932.

[26] R. M. van der Veen, A. Cannizzo, F. van Mourik, A. Vlek Jr, M. Chergui, J. Am. Chem. Soc. 2011, $133,305$.

[27] M. Mohamed, D. Tonti, A. al Salman, M Chergui, ChemPhysChem 2005, 6, 2505.

[28] C. Bonati, M. Mohamed, D. Tonti, G. Zgrablic, S. Haacke, F. van Mourik, M. Chergui, Phys. Rev. B 2005, 71, 205317. 
[29] A. al Salman, A. Tortschanoff, G. van der Zwan, F. van Mourik, M. Chergui, Chem. Phys. 2009, $357,96$.

[30] C. Bonati, A. Cannizzo, D. Tonti, A. Tortschanoff, F. van Mourik, M. Chergui, Phys. Rev. B 2007, 76, 033304.

[31] F. van Mourik, G. Giraud, D. Tonti, M. Chergui, G. van der Zwan, Phys. Rev. B 2008, 77, 165303.

[32] A. Callegari, D. Tonti, M. Chergui, Nano Lett. 2003, 3, 1565.

[33] L. Bonacina, A. Callegari, C. Bonati, F. van Mourik, M. Chergui, Nano Lett. 2006, 6, 7.

[34] A. Cannizzo, O. Braem, G. Zgrablic, A. Tortschanoff, A. A. Oskouei, F. van Mourik, M. Chergui, Optics Lett. 2007, 32, 3555.

[35] E. Portuondo-Campa, A. Tortschanoff, F. van Mourik, M. Chergui, J. Chem. Phys. 2008, 128, 244718.

[36] C. Bressler, R. Abela, M. Chergui, Z. Kristallogr. 2008, 223, 307

[37] C. Bressler, M. Chergui, Annu. Rev. Phys. Chem. 2010, 61, 263.

[38] M. Chergui, A. H. Zewail, ChemPhysChem 2009, 10, 28 .

[39] C. J. Milne, R. M. van der Veen, V.-T. Pham, F. A. Lima, H. Rittmann-Frank, M. Reinhard, S. Karlsson, T. J. Penfold, M. Chergui, Chimia 2011, 65, 303.

[40] W. deBoeij, M. Pshenichnikov, D. Wiersma, Chem. Phys. Lett. 1996, 253, 53.

[41] T. Joo, Y. Jia, J. Yu, M. Lang, G. Fleming, $J$. Chem. Phys. 1996, 104, 6089.

[42] J. Sweetser, D. Fittinghoff, R. Trebino, Optics Lett. 1997, 22, 519

[43] P. Baum, S. Lochbrunner, E. Riedle, Optics Lett. 2004, 29, 1686.

[44] R. Schoenlein, L. Peteanu, R. Mathes, C. Shank, Science 1991, 254, 412.

[45] M. Ponder, R. Mathies, J. Phys. Chem. 1983 $87,5090$.

[46] R. Mathies, L. Stryer, Proc. Nat. Acad. Sci. USA 1976, 73, 2169 .

[47] C. Consani, O. Braem, F. van Mourik, A. Cannizzo, M. Chergui, Chem. Phys. 2011, submitted.
[48] M. Maroncelli, J. Mol. Liquids 1993, 57, 1.

[49] S. K. Pal, A. H. Zewail, Chem. Rev. 2004, 104, 2099.

[50] V.-T. Pham, T. J. Penfold, R. M. van der Veen, F. Lima, A. ElNahhas, S. Johnson, P. Beaud, R. Abela, C. Bressler, I. Tavernelli, C. J. Milne, M Chergui, J. Am. Chem. Soc. 2011, 133, 12740.

[51] D. Zimdars, R. Francis, C. Ferrante, M. Fayer, J. Chem. Phys. 1997, 106, 7498.

[52] D. Zhong, S. Pal, D. Zhang, S. Chan, A. H. Zewail, Proc. Nat. Acad. Sci. USA 2002, 99, 13.

[53] E. F. Aziz, M. H. Rittmann-Frank, K. M. Lange, S. Bonhommeau, M. Chergui, Nat. Chem. 2010 , 2,853 .

[54] W. Gawelda, M. Johnson, F. M. F. de Groot, R. Abela, C. Bressler, M. Chergui, J. Am. Chem. Soc. 2006, 128, 5001.

[55] A. Cannizzo, F. van Mourik, W. Gawelda, G. Zgrablic, C. Bressler, M. Chergui, Angew. Chem. Int. Ed. 2006, 45, 3174.

[56] O. Bräm, F. Messina, A. M. El-Zohry, A Cannizzo, M. Chergui, J. Am. Chem. Soc. 2011 , submitted.

[57] G. Benko, J. Kallioinen, J. E. I. Korppi-Tommola, A. P. Yartsev, V. Sundstrom, J. Am. Chem Soc. 2002, 124, 489

[58] 'Spin Crossover In Transition Metal Compounds II', Eds. P. Gutlich, H. Goodwin, 2004, 233, 1.

[59] 'Spin Crossover In Transition Metal Compounds II', Eds. P. Gutlich, H. Goodwin, 2004, $234,155$.

[60] W. Gawelda, A. Cannizzo, V.-T. Pham, F. van Mourik, C. Bressler, M. Chergui, J. Am. Chem. Soc. 2007, 129, 8199

[61] W. Gawelda, V.-T. Pham, M. Benfatto, Y. Zaushytsin, M. Kaiser, D. Grolimund, S. Johnson, R. Abela, C. Bressler, M. Chergui, Phys. Rev. Lett. 2007, 98, 057401.

[62] C. Bressler, C. Milne, V.-T. Pham, A. ElNahhas, R. M. van der Veen, W. Gawelda, S. Johnson, P. Beaud, D. Grolimund, M. Kaiser, C. N. Borca, G. Ingold, R. Abela, M. Chergui, Science 2009, 323, 489.

[63] R. Bakova, M. Chergui, C. Daniel, A. Vlcek, S. Zalis, Coord. Chem. Rev. 2011, 255, 975.
[64] M. E. Moret, I. Tavernelli, U. Rothlisberger, J. Phys. Chem. B 2009, 113, 7737.

[65] M. E. Moret, I. Tavernelli, M. Chergui, U. Rothlisberger, Chem. Eur. J. 2010, 16, 5889.

[66] R. M. van der Veen, C. J. Milne, A. el Nahhas, F. A. Lima, V.-T. Pham, J. Best, J. A. Weinstein C. N. Borca, R. Abela, C. Bressler, M. Chergui, Angew. Chem. Int. Ed. 2009, 48, 2711.

[67] R. M. van der Veen, A. Cannizzo, J. A Weinstein, M. Chergui, to be published.

[68] C. Bonati, A. Cannizzo, F. van Mourik, M. Chergui, in 'Ultrafast Phenomena in Semiconductors and Nanostructure Materials', Xii, 2008, 6892 , H8920.

[69] D. Tonti, F. van Mourik, M. Chergui, Nano Lett. 2004, 4, 2483.

[70] A. Al Salman, A. Tortschanoff, M. B. Mohamed, D. Tonti, F. van Mourik, M. Chergui, Appl. Phys. Lett. 2007, 90, 093104.

[71] G. V. Hartland, Annu. Rev. Phys. Chem. 2006 $57,403$.

[72] O. Braem, C. Consani, A. Cannizzo, M. Chergui, J. Phys. Chem. A 2011, submitted.

[73] A. A. Oskouei, C. Consani, O. Bräm, A. Tortschanoff, A. Cannizzo, M. Chergui, Chem. Phys. 2011, submitted.

[74] R. M. Hochstrasser, Proc. Nat. Acad. Sci. USA 2007, 104, 14189.

[75] R. M. Hochstrasser, Proc. Nat. Acad. Sci. USA 2007, 104, 14190.

[76] P. Hamm, J. Helbing, J. Bredenbeck, Annu. Rev. Phys. Chem. 2008, 59, 291.

[77] A. Ghosh, R. M. Hochstrasser, Chem. Phys. 2011, doi: 10.1013/chemphys.2011.07.018.

[78] T. Brixner, J. Stenger, H. M. Vaswani, M. Cho, R. E. Blankenship, G. R. Fleming, Nature 2005, 434, 625 .

[79] D. Zigmantas, E. L. Read, T. Mancal, T. Brixner, A. T. Gardiner, R. J. Cogdell, G. R. Fleming, Proc. Nat. Acad. Sci. USA 2006, 103, 12672.

[80] G. S. Schlau-Cohen, A. Ishizaki, G. R. Fleming, Chem. Phys. 2011, 386, 1. 